\title{
Characterising the local void with the X-ray cluster survey REFLEX II
}

\author{
Chris A. Collins ${ }^{1}$, Hans Böhringer ${ }^{2}$, Martyn Bristow ${ }^{1}$ \\ and Gayoung Chon ${ }^{2}$ \\ ${ }^{1}$ Astrophysics Research Institute, Liverpool John Moores University, IC2, Liverpool Science \\ Park, 146 Brownlow Hill, Liverpool L3 5RF, UK \\ email:c.a.collins@ljmu.ac.uk \\ ${ }^{2}$ Max-Planck-Institut für extraterrestrische Physik, D-85748 Garching, Germany \\ email: hxb@mpe.mpg.de
}

\begin{abstract}
Claims of a significant underdensity or void in the density distribution on scales out to $\simeq 300 \mathrm{Mpc}$ have recently been made using samples of galaxies. We present the results of an alternative test of the matter distribution on these scales using clusters of galaxies, which provide an independent and powerful probe of large-scale structure. We study the density distribution of X-ray clusters from the ROSAT-based REFLEX II catalogue, which covers a contiguous area of 4.24 steradians in the southern hempsphere (34\% of the entire sky). Using the normalised comoving number density of clusters we find evidence for an underdensity $(30-40 \%)$, out to $z \sim 0.04$, equivalent to $\simeq 170 \mathrm{Mpc}$ and with a significance of $3.4 \sigma$. On scales between $300 \mathrm{Mpc}$ and $1 \mathrm{Gpc}$ the distribution of REFLEX II clusters is consistent with being uniform. We also confirm recent results that the underdensity has a large contribution from the direction of the South Galactic Cap region, but is not significant in the direction of the Northern Galactic Cap as viewed from the southern sky. Both the limited size of the detected underdensity and its lack of isotropy, argue against the idea that the Type Ia supernovae data can be explained without the need for dark energy.
\end{abstract}

Keywords. galaxies: clusters, cosmology: large-scale structure of universe, general, X-rays: galaxies: clusters

\section{Introduction}

There is significant interest in the possibility that we live close to the centre of a large isotropic void that can mimic an accelerating universe, as revelaed by the observations of supernovae (Schmidt et al. 1998; Perlmutter et al. 1999), without the need for dark energy (e.g., Alexander et al. 2009; February et al. 2010). In these "minimal-void" scenarios an underdensity of $\sim 40 \%$ stretching to $\sim 300 \mathrm{Mpc}$ or more can reproduce both the supernovae data and other cosmological constraints without the requirement of an accelerating universe, although how difficult it is for these models not to fail at least one cosmological test remains an open question (e.g., Moss et al. 2011). A large void could also explain the tension between measurements of the Hubble constant $\left(H_{0}=67.3 \pm 1.2 \mathrm{~km} \mathrm{~s}^{-1} \mathrm{Mpc}^{-1}\right)$ determined from PLANCK measurements of the cosmic microwave background (Planck Collaboration XVI, 2013) and a higher value $\left(H_{0}=73.8 \pm 2.4 \mathrm{~km} \mathrm{~s}^{-1} \mathrm{Mpc}^{-1}\right)$ based on galaxies hosting both Type Ia supernovae and Cepheid variable stars (Riess et al. 2011). Furthermore, the minimum size of an underdensity large enough to embed the local $H_{0}$ measurements and deep enough to induce a $9 \%$ difference in $H_{0}$ compared to the global mean, a so called "Hubble bubble", is unlikely to occur naturally from density fluctuations in the standard $\Lambda$ CDM model. If substantiated, a cosmology beyond the standard model may be required (Marra et al. 2013). 
Two recent observational studies using K-band selected galaxy samples probe the density distribution on scales 300-400 Mpc. Whitbourn \& Shanks (2014) use the redshifts of 250,000 galaxies compiled from 6dFGS and SDSS to examine the density distribution in three regions covering a total area of 9,000 $\mathrm{deg}^{2}$. They find a range of underdensities from $4-40 \%$ to a depth of $\sim 200 \mathrm{Mpc}$, with the most prominent underdensity concentrated on the South Galactic Cap region of the southern sky. Keenan et al. (2013) use a sample of 35,000 galaxies with redshifts, covering $600 \mathrm{deg}^{2}$, based on the UKIDSS and 2MASS surveys. By fitting the lumonisity function to the galaxy magnitudes the authors provide evidence for $\mathrm{a} \simeq 50 \%$ reduction in the mass density within a volume of $z=0.07$ or about $300 \mathrm{Mpc}$. If representative, these results could have significant implications.

\section{The Data: REFLEX II}

An alternative probe of the underdensity is to use clusters of galaxies. These are well known to be a reliable tracers of the large-scale structure in the universe (e.g., Kaiser 1984) and they easily probe the large volumes necessary to examine the claims of underdensities up to Gpc scales. Furthermore, clusters are biased tracers of the mass distribution compared to galaxies and therefore should amplify any putative density contrast. The study here uses the homogeneous X-ray flux limited cluster survey REFLEX II (Böhringer et al. 2013), which covers a contiguous area of $\simeq 4.24$ steradians in the southern sky below a declination of $+2.5^{0}$ and with a galactic latitide $\left|b_{I I}\right| \geqslant 20^{0}$. REFLEX II is based on the RASS X-ray source detections (Trümper 1993; Voges et al.1999). The nominal flux limit of $1.8 \times 10^{-12} \mathrm{erg} \mathrm{cm}^{-2} \mathrm{~s}^{-1}$ in the energy range $0.1-2.4 \mathrm{keV}$ is reached for $80 \%$ of the survey area, with higher flux limits in the remaining $20 \%$ which have low exposure or high interstellar absorption. The REFLEX II selection function is fully accounted for in the analysis described below. In total there are 913 clusters and groups in REFLEX II above an X-ray luminosity of $L_{X} \geqslant 10^{42} \mathrm{erg} \mathrm{s}^{-1}(0.1-2.4 \mathrm{keV})$; 206 of these lie within $z=0.06$ and 416 out to $z=0.1$. For details of the properties of REFLEX II and how it was constructed see Böhringer et al. (2013). In this paper we adopt $H_{0}=70 \mathrm{~km} \mathrm{~s}^{-1} \mathrm{Mpc}^{-1}$ and $\Omega_{m}=0.3$ with a flat $\Lambda$ CDM cosmology.

\section{Results}

Here we give an overview of our methodology and results. Full details and discussion can be found in Böhringer et al. (2014b). We calculate the relative density distributions for REFLEX II using a best-fit Schechter form for the luminosity function (see Böhringer et al. 2014) in order to predict the number of X-ray clusters with redshift and then divide the number of observed clusters in redshift shells by the predicted value. In Figure 1 we show the normalised comoving density of REFLEX II clusters as a function of redshift. There is a clear cluster underdensity of $30-40 \%$ in the redshift range below $z=0.04$, equivalent to about $170 \mathrm{Mpc}$. Table 1 shows the cumulative ratio of the observed to predicted number of clusters, along with the statistical uncertainty using the best-fit Schechter function above the luminosity limit $L_{X}=10^{42} \mathrm{erg} \mathrm{s}^{-1}(0.1-2.4 \mathrm{keV})$. The overall significance of the underdensity averaged over the entire southern sky out to $z \simeq 0.04$ is $3.4 \sigma$. The void is still present at a significant level if the minimum luminosity limit is increased by a factor 20 to $L_{X} \geqslant 2 \times 10^{43} \mathrm{erg} \mathrm{s}^{-1}(0.1-2.4 \mathrm{keV})$ indicating that both richer clusters and poorer groups follow the same density pattern. More specifically the relative density distributions for REFLEX II volume-limited subsamples over the redshift range $z=0.015$ to $z=0.15$ show remarkably similar density ratios in overlapping redshift shells (see Böhringer et al.2014b). We also note that there 


\begin{tabular}{|c|c|c|c|c|c|c|c|}
\hline$z$ & 0.02 & 0.03 & 0.04 & 0.05 & 0.06 & 0.07 & 0.08 \\
\hline Ratio $\mid$ & $0.63 \pm 0.11$ & $0.53 \pm 0.07$ & $0.61 \pm 0.07$ & $0.71 \pm 0.06$ & $0.81 \pm 0.05$ & $0.88 \pm 0.06$ & $0.93 \pm 0.05$ \\
\hline
\end{tabular}

Table 1. Cumulative ratio of observed to predicted cluster numbers with redshift.

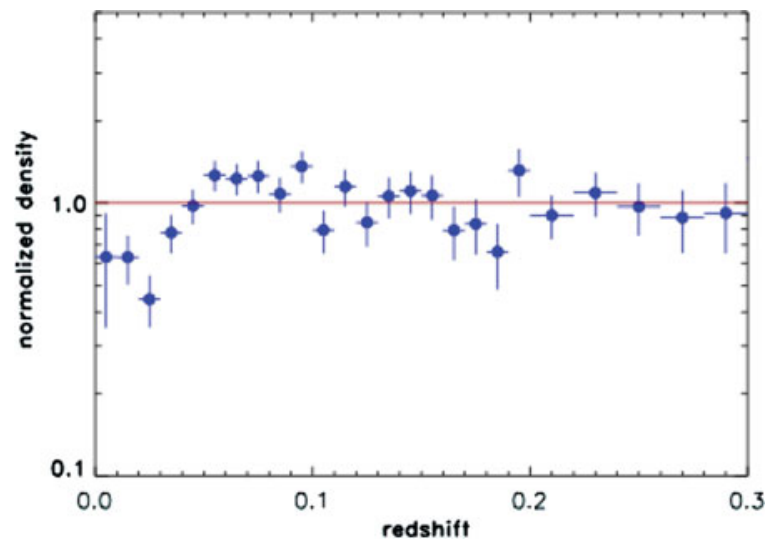

Figure 1. Normalised cluster density distribution from REFLEX II as a function of redshift using a minimum X-ray luminosity of $10^{42} \mathrm{erg} \mathrm{s}^{-1}(0.1-2.4 \mathrm{keV})$. There is a clear signal of an underdensity within a radius of about $z=0.04$, corresponding to $\simeq 170 \mathrm{Mpc}$.

is significant overdensity detected at redshifts between $z=0.05-0.08$. This is due to the dominance of superclusters such as Shapley $(z=0.046)$ and superclusters 42 (part of the Horologium-Reticulum supercluster at $z=0.065)$ and 62 identified in REFLEX II by Chon et al. (2013) - see also the contribution by Chon et al. in this volume.

Next we restrict our analysis to the identified regions at the South Galactic Cap (SGC) and North Galactic Cap (NGC) where Whitbourn \& Shanks (2014) carried out their underdensity analysis using 6dFGS galaxies, as mentioned in Section 1. In Figure 2 we show the normalised comoving density of REFLEX II clusters as a function of redshift for both the SGC and NGC regions, along with the galaxy results from Whitbourn \& Shanks (2014). Whereas at the SGC the density falls to $0.45 \pm 0.10$ at $z=0.05(0.35 \pm 0.10$ at $z=0.04)$, at the NGC the density is $1.02 \pm 0.17$ at $z=0.05(0.83 \pm 0.18$ at $z=0.04)$. This indicates that the detected $30-40 \%$ underdensity from the full REFLEX II area is not isotropic. In Table 2 we show the comparison of the ratio of observed to predicted numbers of clusters within $z=0.05$ and $z=0.1$, providing a direct comparison with Whitbourn \& Shanks (2014). Overall there is excellent agreement between the two surveys in both the SGC and NGC regions, even accounting for the relative biasing (see Section 4) and despite the presence of relatively large uncertainties, which reassuringly indicates that both galaxies and clusters are following the same local density variations.

\section{Implications}

The detection of an overall underdensity of $40 \pm 15 \%$ in the cluster distribution implies an underdensity in the matter distribution of $15 \pm 5 \%$, based on an average bias of 2.5-3.0, appropriate for REFLEX II clusters at low redshift (Chon et al. 2014). In turn this implies a $3 \pm 1 \%$ larger Hubble constant locally, based on simple linear theory. This is not sufficient on its own to explain the tension between the local and distant determinations of the Hubble constant which differ by $9 \%$ as discussed in Section 1. Furthermore the scale of our detected void falls short of the $\simeq 300 \mathrm{Mpc}(z=0.007)$ required to explain supernovae data 


\begin{tabular}{|c|c|c|c|c|}
\hline & \multicolumn{2}{|c|}{$z<0.05$} & \multicolumn{2}{|c|}{$z<0.1$} \\
\hline Region & SGC & NGC & SGC & NGC \\
\hline REFLEX II & $0.45 \pm 0.10$ & $1.02 \pm 0.17$ & $0.84 \pm 0.09$ & $1.18 \pm 0.12$ \\
\hline W\&S & $0.60 \pm 0.05$ & $0.96 \pm 0.10$ & $0.75 \pm 0.05$ & $0.94 \pm 0.07 \mid$ \\
\hline
\end{tabular}

Table 2. Relative densities from REFLEX II along with the results of Whitbourn \& Shanks 2014. The redshifts $z=0.05$ and 0.1 are chosen to enable a direct comparison.
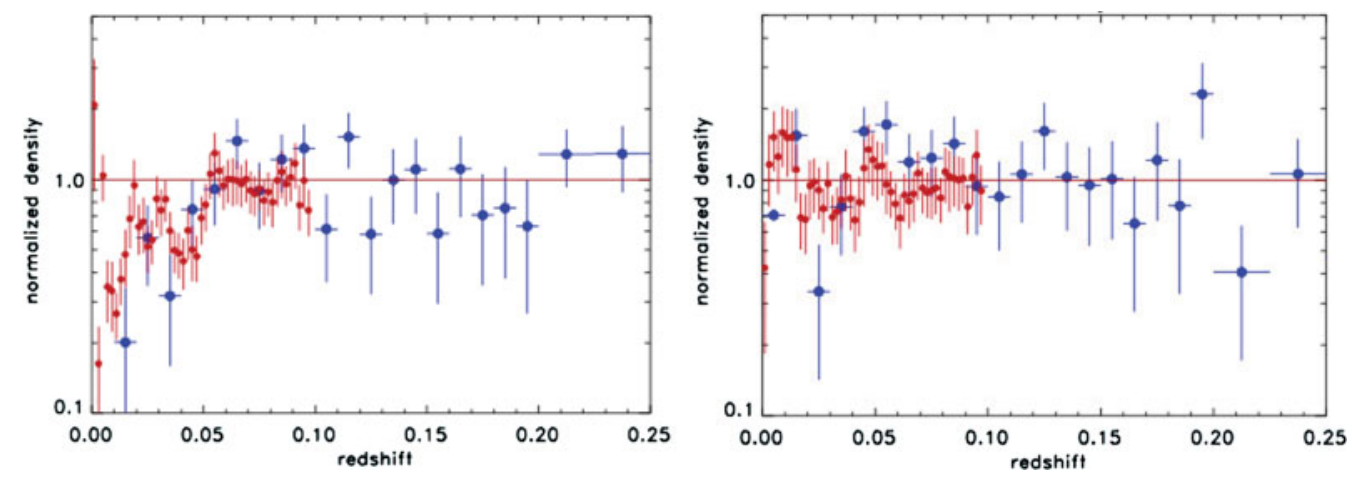

Figure 2. Density distributions for REFLEX II with the galaxy study (smaller points) by Whitbourn \& Shanks (2014) for the 6dFGS regions: SGC (left) and NGC (right).

without dark energy induced acceleration. An examination of Figure 1 reveals that from $300 \mathrm{Mpc}$ up to $1 \mathrm{Gpc}(z=0.25)$ the density distribution of REFLEX II is exceptionally uniform. The limited size of the measured underdensity and its lack of isotropy across the southern sky are in contrast to models which place us at the centre of a large void.

\section{References}

Alexander, S., Biswas, T.,Notari, A., \& Vaid, D. 2009, JCAP, 9, 25

Böhringer, H., Chon, G., Collins, C. A., Guzzo, L., et al. 2013, A\&\&A, 555, A30

Böhringer, H., Chon, G., \& Collins. C. A. 2014, A\&A, 570, A31

Böhringer, H., Chon, G., Bristow, M., \& Collins, C. A. 2014b, A\& A, in press, arXiv:1410.2172

Chon, G., Böhringer, H., \& Nowak, N. 2013, MNRAS, 429, 3272.

Chon, G., Böhringer, H., Collins, C. A., \& Krause, M. 2014, A\&A, 567, A144

February, S., Larena, J., Smith, M., \& Clarkson, C. 2010, MNRAS, 405, 2231

Kaiser, N. 1984, ApJ, 284, L9

Keenan, R. C., Barger, A. J., \& Cowie, L. L. 2013, ApJ, 775, 62

Marra, L., Amendola, L., Sawicki, I., \& Valkenburg, W. 2013, Phys. Rev. Lett., 110, 241305

Moss, A., Zibin, J. P., \& Scott, D. 2011, Phys. Rev. D, 83, 103515

Perlmutter, S., Aldering, G., Goldhaber, G., Knop, R. A., et al. 1999, ApJ 517, 565

Planck Collaboration 2013 results XVI 2013, arXiv 1303.5076v2

Riess, A. G., Macri, L., Casertano, S.,Lampeitl, H., et al. 2011, ApJ, 730, 119

Schmidt, B., Suntzeff, N. B., Phillips, M. M., Schommer, R. A., et al. 1998, ApJ, 507, 46

Trümper, J. 1993, Science, 260, 1769

Voges, W., Aschenbach, B., Boller, T., Bräuninger, H., et al. 1999, A\&A, 349, 389

Whitbourn, J. R. \& Shanks, T. 2014, MNRAS, 437, 2146 